\title{
Increasing Exercise Work Capacity via Nutritional Intervention by Administration of Mitochondrial Augmentation Therapy (MAT) Associated Nutrients
}

\author{
Constanta Badulescu ${ }^{1}$ GDIP Kin, Certified Kinesiologist, Shuk Yee Ngan $^{2}$ Dipl BTech, BSc, Kamilia \\ Talipova $^{2}$ Dipl BTech, BSc and Frank Merante ${ }^{2 *}$ MSc, PhD \\ ${ }^{1}$ The Karate Lab, Toronto Ontario Canada \\ ${ }^{2}$ Seneca College, School of Biological Sciences and Applied Chemistry, Toronto, Ontario Canada M3J 3M6
}

*Corresponding author: Frank Merante, Seneca College, School of Biological Sciences and Applied Chemistry, Toronto, Ontario Canada M3J 3M6, Canada

\section{ARTICLE INFO}

Received: 幽 November 19, 2019

Published: 幽 December 09, 2019

Citation: Constanta Badulescu GDIP Kin, Certified Kinesiologist, Shuk Yee Ngan Dipl BTech, BSc, Kamilia Talipova Dipl BTech, BSc, Frank Merante MSc, PhD. Increasing Exercise Work Capacity via Nutritional Intervention by Administration of Mitochondrial Augmentation Therapy (MAT) Associated Nutrients. Biomed J Sci \& Tech Res 23(4)-2019. BJSTR. MS.ID.003944.

Keywords: Nutritional Intervention; Mitochondria; Athletic Performance; Recovery; Aerobic Metabolism; Krebs's Cycle; Respiratory Chain; Delayed Onset Muscle Soreness (DOMS); Malic Acid; Citric Acid; Vitamin C; Pyruvic Acid; Supplements; Mitochondrial Augmentation Therapy (MAT)

\section{ABSTRACT}

Background: Physical exertion places a demand on cellular metabolic reserves. By providing key metabolic intermediates to mitochondria before, during and after training, optimal cellular homeostasis and metabolic replenishing can be augmented, enabling optimal maintenance and recovery of cellular reserves aimed at improving aerobic cellular function. The purpose of this pilot study was to evaluate if the exercise work capacity and recovery can be augmented through the administration of a synergistic blend of key metabolic intermediates which included: Citrate, Malate, Pyruvate and Vitamin C.

Methods: A total of 14 participants volunteered to complete two identical training sessions; a Control session and a Supplemented session two weeks later. Three identical exercises, comprising 10 sets each, were included in the two sessions which included a 20 second work-set followed, by 90 seconds of rest. The number of repetitions, the repetition in which muscle fatigue and exercise induced muscle pain (burn) initiated, and peak muscle pain (burn) severity were recorded.

Results: Nutritional intervention using tricarboxylic cycle intermediates provides a means for directly modulating mitochondrial performance expressed physiologically as improved work capacity and the ability to augment athletic performance. Concordantly, this study demonstrated improved recovery post-training minimizing delayed onset muscle soreness (DOMS).

Abbreviations: MAT: Mitochondrial Augmentation Therapy; DOMS: Delayed Onset Muscle Soreness; 1RM: one (1)-repetition maximum; TCAI: Tricarboxylic Acid Intermediates; BDP: Badulescu Deficit-Training Protocol; PBS: Peak Burn Severity; EWC: Exercise Work Capacity; SEM: Standard Error Of The Mean.

\section{Introduction}

Physical and mental exertion places a demand on cellular metabolic reserves. Numerous supplements, including proteinaceous compounds such as whey protein isolate, casein or individual amino acids have been used in attempts to improve and hasten recovery in athletes particularly post- workout with mixed results. Although beneficial, these components aim to repair or augment muscle myofiber density and assist in the repair of exercise induced

muscle damage [1-4] and only indirectly aid mitochondrial support and recovery or improved aerobic function. The mitochondria are responsible for a multitude of biochemical functions, one of the most important being cellular energy production for sustained cellular metabolism and subsequent recovery from aerobically driven cellular demands. In addition to being the powerhouse for cells, many of the biochemical reactions that take place within the mitochondria are directly responsible for producing the plethora of 
metabolic intermediates required to make amino acids and other key anaplerotic biochemical components, many of which are absolutely required, and necessary, for fostering muscle growth and repair. Collectively, these metabolic components are responsible for limiting or reduced delayed onset muscle soreness (DOMS) and increasing strength, endurance and power [5]. By providing key metabolic intermediates for mitochondrial assimilation before, during and after training, may enable optimal cellular homeostasis and provide metabolic replenishing molecules to enable optimal recovery of cellular reserves and maintain aerobic cellular function.

A number of different agents have been suggested to improve or augment performance and mitochondrial function in athletes. These include, but are not limited to supplementary glucose, various amino acids [6-8] (i.e., L- arginine [9], beta-alanine [10,11], citrulline (in the form of citrulline-malate $[12,13]$ or citrulline alone $[12,14,15]$, glutamine [3,16], taurine [17] among other amino acids [18] and/ or a combination of branched-chain amino acids, particularly including L- Leucine [19]. Additional ergogenic and anaplerotic agents have been investigated, including [20-22], pyruvate [23-25], citrate [26-28], or malate [29] alone, creatine [22,30,31] (often in the form of pyruvate-creatine or creatine citrate [32,33], carnitine $[34,35]$, various vitamins [36,37], co-factors [38], and antioxidants $[39,40]$, among many others, particularly plant derived extracts or natural products (for example green tea [41], Cordyceps sinensis and yohimbine [42]) or complex derivatives thereof concocted into fortified beverages with supplementary carbohydrates [28,43] or protein isolates [44]. Additional investigations have explored the augmentation of athletic performance by specifically boosting nitric oxide levels via supplementation with L-arginine $[20,45]$, or indirectly by using an arginine precursor such as citrulline [17] which has been shown to extend time to exhaustion [46]. The beneficial role of these agents has typically been investigated using high dosages of a single agent and defined exercise routines.

Endurance and recovery as exemplified by reduced DOMS has been shown to be improved by supplementing with creatine. Creatine boosts intracellular phosphor-creatine levels and can act as an energy reserve compound improving athletic performance $[33,47]$ when provided at therapeutically significant doses, but may contribute to increased fat and triglyceride levels in supplemented athletes [48]. Similarly, branched-chain amino acids have been shown to improve performance and recovery [49], but not necessarily exercise induced muscle damage [7]. To directly target mitochondria, various individual metabolic intermediates have been tested with varying degrees of success and this mitochondrial augmentation therapy [MAT] has been explored for the improvement of syndromes associated with critical illness, disease states, septic shock fostering mitochondrial resuscitation [50-52]. There remains a need for improved compositions and methods for improving mitochondrial function and post-exertion recovery. This investigation examines the synergistic combination of key metabolic intermediates into a blend to permit direct nutritional intervention aimed at improving exercise capacity and recovery. This blend included: Citrate, malate and pyruvate in the presence of the cellular antioxidant and potential carnitine cofactor, ascorbate (Vitamin C), to provide an effective combination for athletic support, improved exercise work capacity and recovery.

\section{Methods}

\section{Participants}

The research activities were approved by Seneca College's Research Ethics Review Board File \#16-2. Fourteen healthy individuals (4 men and 10 women) volunteered to participate in this study. Participants ranged in age from 18-61 years old, all physically active and healthy, free of cardiovascular disease or major orthopedic conditions that would limit their abilities to perform the two structured weight training sessions. All subjects, had different levels of cumulative strength training experience, participated in a of minimum two cross training sessions per week for at least two years, and managed to complete all the planned activities of this study. All individuals were experienced in executing the proper technique of the exercises prescribed during the protocol. Each participant was familiar with their one (1) -repetition maximum (1RM). Weights were chosen for everyone based on their selfreported 1RM. Prior to the first session, each person subjected to the protocol, was required to complete a questionnaire describing their fitness goals, training experience and nutritional habits (diet and possible use of supplements).

\section{Experimental Design}

\section{Orientation and Informed Consent}

Prior to the first exercise session, participants were given an orientation session. The volunteers were informed about the scope of this study: the investigation of physical performance improvement and the possible metabolic effects with the use of a Health Canada approved natural supplement (NPN80070757) containing citrate, malate, pyruvate and ascorbate. The structure of the workout and the testing methodologies were disclosed to the participants. An informed consent form / invitation to participate form was provided to the volunteers. This pilot study was comprised of two phases: The Baseline (the Control Phase) and the Supplemented session (Supplemented Phase). After obtaining face-to-face consent, the participants were instructed to warm up. The pre-exercise preparation consisted of jogging for five minutes on a treadmill at $5-6 \mathrm{mph}$, followed by a five minutes circuit of 10 squats, 10 lunges, and 5 push-ups at a moderate pace with 45 seconds rest in between. The participants (Table 1) were asked to perform physical structured exercise custom developed for this study and termed the Badulescu Deficit-Training Protocol (BDP).

Table 1: Characteristics of Male and Female Participants.

\begin{tabular}{|c|c|c|}
\hline Variable & Men & Women \\
\hline No & 4 & 10 \\
\hline Age (average) & 37 & 43.7 \\
\hline Training (Av h/week) & 3.5 & 4 \\
\hline
\end{tabular}


The BDP design was aimed to have the participants perform at a high intensity level with anaerobically generated energy. The contribution of the fast-glycolytic system increases rapidly after the 10 seconds of intense exercise. Thus, a greater aerobic demand response was created and some of the fatigue was carried into the subsequent sets, participants experiencing exercise induced muscle pain severity and its onset at different stages as the workout progressed. The protocol consisted of three sections. Each one included one exercise performed for 10 sets of 20 seconds maximal effort work and 90 second break (Figure 1). The first section consisted of squats aiming to have an average load of $70 \%$ of body weight (BW) and $60 \%$ of 1 RM (Table 2). Deadlifts were performed in the second section using an identical interval structure. The load used was an average of $70 \%$ of BW and $60 \%$ of 1 RM. The last part was the floor press exercise. The structure of work sets, and break time was kept, and the weight used was of an average $45 \%$ of BW and $45 \%$ of 1 RM.

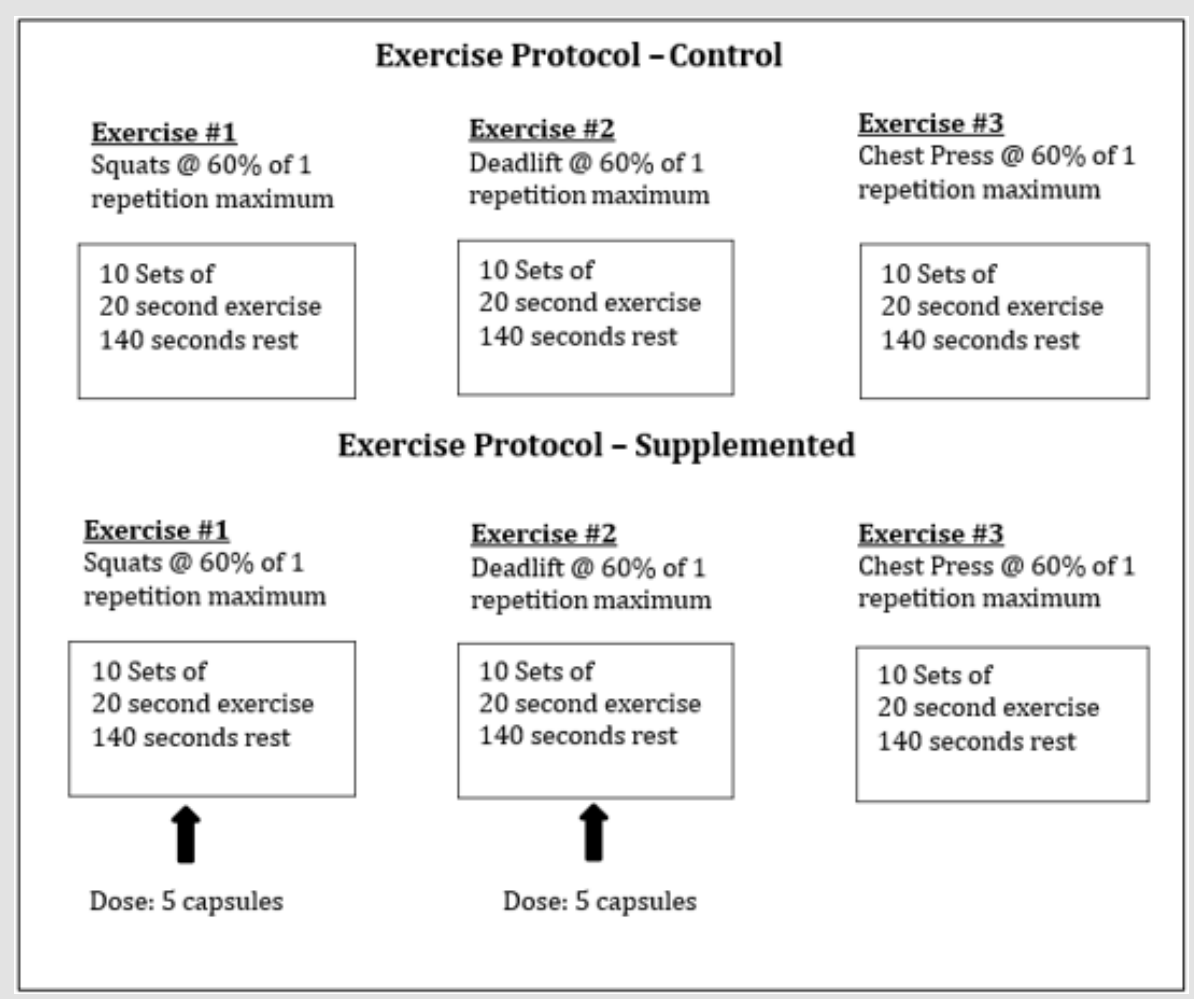

Figure 1: Exercise protocol for Control and Supplemented testing conditions.

Table 2: Percentage of One Repetition Maximum Weight.

\begin{tabular}{|c|c|c|c|c|}
\hline Exercise & M \%BW & M \%1RM & W \%BW & W \%1RM \\
\hline Squats & 65.20 & 55 & 75.9 & 64.2 \\
\hline Deadlifts & 67 & 52 & 75.9 & 67 \\
\hline Floor press & 43.8 & 48.05 & 45.6 & 47.28 \\
\hline
\end{tabular}

Kinesiology Data Collection: Four parameters were recorded and subsequently used in assessing the potential improvement between the Control and Supplemented sessions:

1. Repetitions performed during each set

2. Onset of burn (the moment the individual started to feel "muscle pain")

3. Muscle pain (Burn) severity using a pain scale:

a. 1 - No Burn, "at rest early effort"

b. 2-ONSET, "1st feeling of pain"

c. 3-MILD, "mild, form maintained" d. 4 - MODERATE, "it hurts, form impaired"

e. $\quad 5$ - SEVERE "pain unbearable”, "form break down", "must stop"

4. A post-exercise recovery (over a period of 7 days with the same 1 to 5 pain scale).

\section{Dosing}

The volunteers who participated in this study supplemented their dietary regime with a Health Canada approved Natural Health Supplement (NPN 80070757) and were assessed before and after their consumption. The conditions of the supplement usage were in accordance with Health Canada Guidance. The supplement was 
used as per the product label. One week following the Control Session, the participants were instructed to take the supplement dispersed throughout the day, before and after their workouts. Smaller athletes $(\leq 68 \mathrm{~kg}$ ) took up to 8 capsules a day: 2 in the morning before breakfast, 3 prior to exercise and 3 post exercise. Larger athletes ( $\geq 68 \mathrm{~kg}$ ) took up to 10 capsules a day: 4 in the morning before breakfast, 3 before their workouts followed by other 3 capsules. The morning of the session, the subjects took two capsules. Before the Supplemented session, 2 capsules were administered, and the same quantity was maintained throughout. After the first section of BDP, another dose was given to the volunteers. The supplement was taken next after deadlifts part. At the end of the workout the participants were administered another dose.

\section{Rest Period}

Between the Control and Supplemented sessions, there was a two weeks rest interval. This was considered an active rest period, trying to simulate real life conditions of an athlete training regimen. The participants were able to participate in the same three group classes during the first week and only two group classes the second week. The third session of the second week was the BDP workout. The post exercise soreness logs were filled out after both sessions, using the 1 to 5 -pain scale.

\section{Statistical Analysis}

The mean values, standard deviation and standard error of the mean (SEM) were calculated for the Control exercise results vs. the Supplemented exercise results. This was performed in four groups:

\section{Group 1. The Aggregate of all sets-Control vs. Supplemented:}

a. Comparison of average volume of repetitions completed per set - Control vs. Supplemented.

b. Comparison of average volume of repetitions completed vs. the average volume of repetitions completed with no burn Control vs. Supplemented.

c. Comparison of average peak burn severity - Control vs. Supplemented.

\section{Group 2. Squat sets - Control vs. Supplemented:}

a. Comparison of average volume of repetitions completed per set - Control vs. Supplemented.

b. Comparison of average volume of repetitions completed vs. the average volume of repetitions completed with no burn Control vs. Supplemented.

c. Comparison of average peak burn severity - Control vs. Supplemented.

\section{Group 3. Deadlift sets - Control vs. Supplemented:}

a. Comparison of average volume of repetitions completed per set - Control vs. Supplemented. b. Comparison of average volume of repetitions completed vs. the average volume of repetitions completed with no burn Control vs. Supplemented.

c. Comparison of average peak burn severity - Control vs. Supplemented.

\section{Group 4. Floor press sets - Control vs. Supplemented:}

a. Comparison of average volume of repetitions completed per set - Control vs. Supplemented.

b. Comparison of average volume of repetitions completed vs. the average volume of repetitions completed with no burn Control vs. Supplemented.

c. Comparison of average peak burn severity - Control vs. Supplemented.

A two-tailed paired $t$ test was used to determine if the changes observed between the various means of the Control and Supplemented group were significant. The analysis was completed using Microsoft Excel. Significance for p-values was determined using an alpha level of $\left(^{*}\right) \mathrm{p}<0.05$.

\section{Results}

Exercise Work Capacity (EWC) in Control vs. Supplemented conditions was analyzed from three different perspectives. EWC as represented by:

1. The number of repetitions completed,

2. The repetition at which athletes experienced the onset of exercise induced muscle pain (Onset of Burn), and

3. The Peak Burn Severity (PBS) experienced by athletes at the conclusion of each work set.

\section{EWC - Repetitions Completed}

The results indicated that nutritional intervention created a significant positive impact on EWC in terms of repetitions completed for the Supplemented conditions, as the total number of repetitions completed increased in the Supplemented condition vs. Control. The increase was observed across all exercises (Table 3); ( $p \leq 0.05$ ) and a mean increase of greater than one repetition per set with the supplemented condition (Figures $2 \& 3$ \& Table 4) p $\leq 0.05$ ). When regarding the variance in aggregated repetitions completed across all participants, out of the 420 sets assessed, in the Supplemented condition, 239 (57\%) sets had an increase in the number of repetitions, $90(21 \%)$ sets had a decrease, while 91 (22\%) sets presented no change. When assessing the individual exercise sets performed, the variance in the squat for the number of repetitions completed between the Control and Supplemented conditions showed that out of the 140 sets performed, the Supplemented condition exhibited an increase of 85 (61\%) repetitions; 26 (19\%) sets had a decrease number of repetitions, while 29 (21\%) sets presented no change in repetition number. Concordantly, the set 
variance in the deadlift showed that out of the 140 sets assessed, for the Supplemented condition 77 (55\%) had an increase in the number of repetitions, 41 (29\%) had a decrease, while 22 (16\%) sets presented no change. Finally, for the floor press $85(61 \%)$ had an increase in the number of repetitions, 32 (23\%) sets had a decrease, while $23(16 \%)$ sets presented no change.

Table 3: Total Count of Repetitions Completed in the Control Condition vs. the Supplemented Condition.

\begin{tabular}{|c|c|c|c|}
\hline & Control & Supplemented & $\begin{array}{c}\boldsymbol{\Delta} \text { Change } \\
\text { (Supplemented }- \text { Control) } * \mathbf{p} \leq \mathbf{0 . 0 5}\end{array}$ \\
\hline Aggregate & 4942 & 5429 & 129 \\
\hline Squat & 1348 & 1477 & 81 \\
\hline Deadlift & 1371 & 2500 & 277 \\
\hline Floor Press & 2223 & & \\
\hline
\end{tabular}

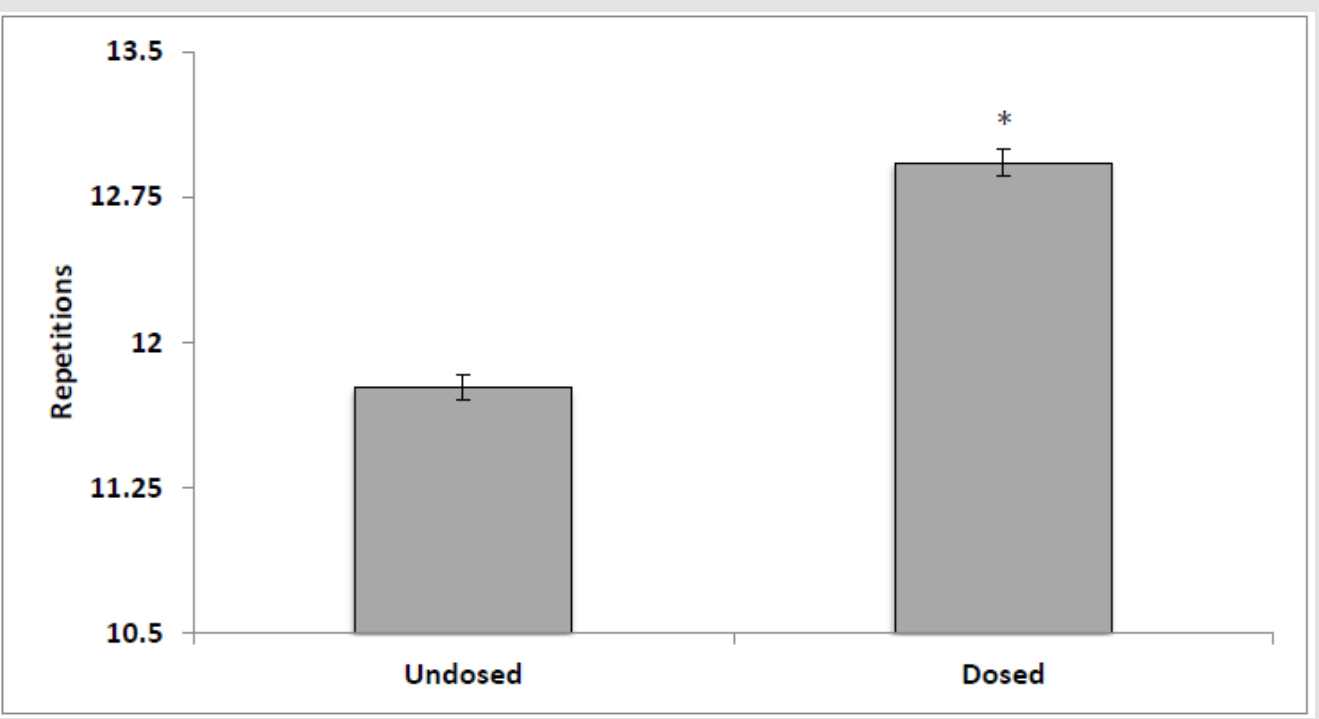

Figure 2: Mean change per athlete in number of repetitions completed increased by $1.16 \pm 0.23$ in the Supplemented condition vs. the Control condition. Significantly different $\left({ }^{*} \mathrm{p}<0.01\right.$ ). Control mean 11.77, SD is 4.67; SEM (shown on figure) is 0.07 . Mean repetitions completed in the Supplemented condition were 12.93, SD 4.98; SEM (shown on figure) is 0.07 . See Table 4 for a comparison of the mean in each of the conditions, Control vs. Supplemented, for squat, deadlift, and floor press.

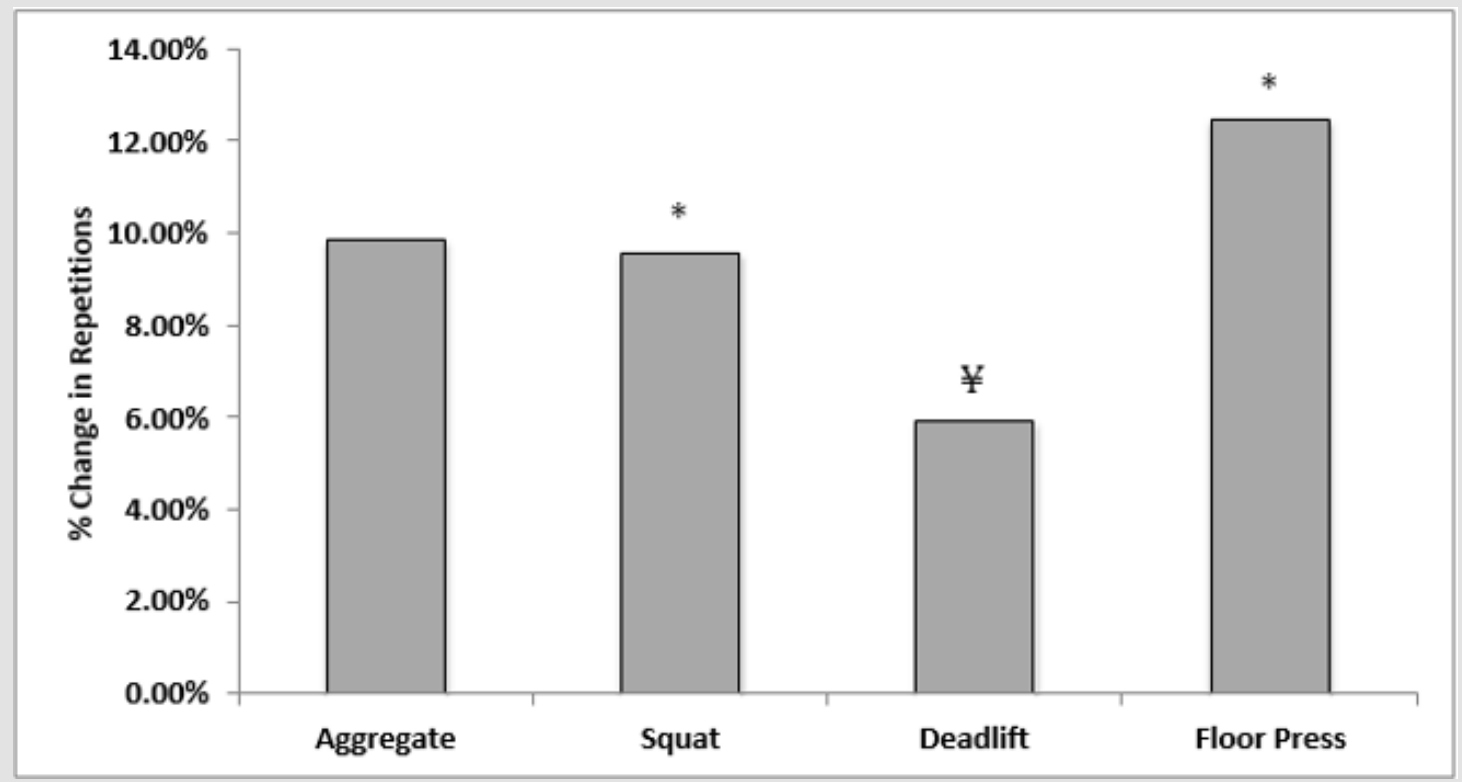

Figure 3: Percent (\%) increase in mean repetitions completed in the Supplemented condition vs. the Control condition. Change is significant. For $\mathrm{p}$ values see Table 4 and Figure 2. 
Table 4: "SD" is the Standard Deviation (Excel st dev. p), "SEM" is the Standard Error of the Mean. The change is significant (Errors reflect the $95 \%$ confidence interval, $z=1.96, ¥ p<0.05)$.

\begin{tabular}{|c|c|c|c|c|}
\hline \multicolumn{5}{|c|}{ Mean Number of Repetitions Completed in the Control Condition vs. the Supplemented Condition } \\
\hline & Mean Control Repetitions (SD, SEM) & Mean Supplemented Repetitions (SD, SEM) & Change & p \\
\hline Squat & $9.63(1.66,0.05)$ & $10.55(1.98,0.05)$ & $+1.84 \pm .27$ & $<0.01$ \\
\hline Deadlift & $9.79(2.58,0.07)$ & $10.37(2.19,0.06)$ & $+0.58 \pm .27$ \\
\hline Floor Press & $15.88(5.54,0.12)$ & $17.86(5.41,0.11)$ & +0.05 \\
\hline
\end{tabular}

\section{EWC - Onset of Muscle Burn}

There appears to be a significant positive impact in EWC in terms of delayed onset of exercise associated muscle pain (burn) in Supplemented conditions as assessed by the variance in aggregate repetitions completed at which athletes reported the onset of burn between the Control and Supplemented condition (Table 5 \& Figure $4 \& 5)$. Out of the 420 sets completed, in the Supplemented condition, $254(60 \%)$ sets had a delay in the onset of burn during the sets completed, $113(27 \%)$ sets presented a hasted onset of burn, while $53(13 \%)$ sets presented no change in the onset of burn moment.

Table 5: "SD" is the Standard Deviation, "SEM" is the Standard Error of the Mean.

\begin{tabular}{|c|c|c|c|c|}
\hline \multicolumn{5}{|c|}{ Change in the Onset of Muscle pain in Control vs. Supplemented Athletes } \\
\hline & $\begin{array}{c}\text { Control Repetitions Without Burn (SD, } \\
\text { SEM) }\end{array}$ & $\begin{array}{l}\text { Supplemented Repetitions Without Burn (SD, } \\
\text { SEM) }\end{array}$ & Change & $\mathbf{p}$ \\
\hline Squat & $6.75(3.04,0.10)$ & $7.56(3.19,0.10)$ & $0.81 \pm .42$ & $<0.03$ \\
\hline Deadlift & $6.79(4.16,0.14)$ & $7.79(3.78,0.11)$ & $1.00 \pm .48$ & $<0.03$ \\
\hline Floor Press & $11.22(6.18,0.16)$ & $13.64(6.18,0.14)$ & $2.42 \pm .65$ & $<0.01$ \\
\hline
\end{tabular}

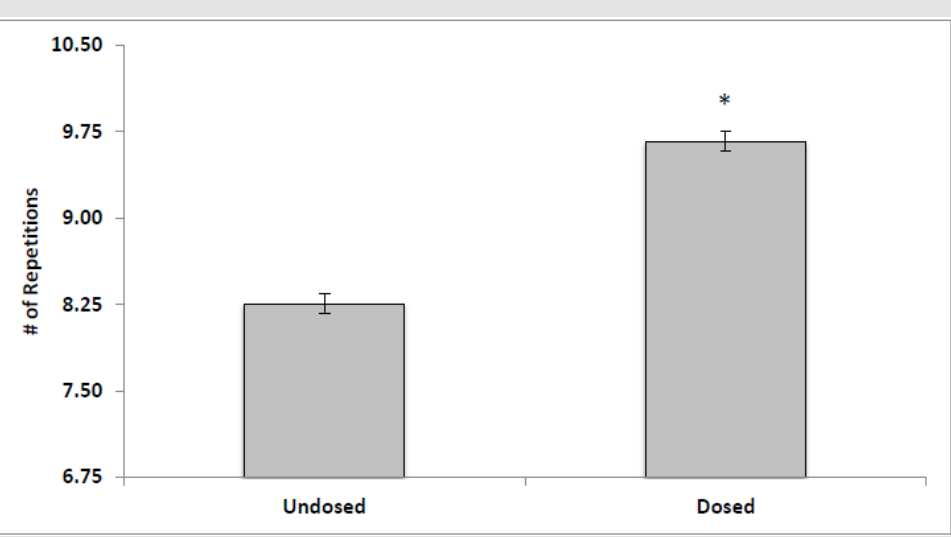

Figure 4: Mean change in the onset of burn increased by $1.41 \pm .31$ (95\% confidence interval, $z=1.96)$ in the Supplemented condition vs. the Control condition. Significantly different $\left({ }^{*} p<0.01\right)$. Control mean of repetitions completed without burn 8.25 , SD (Excel stdev.p) is 5.10; SEM (shown on figure) is 0.09. Mean of repetitions completed without burn in the Supplemented condition was 9.67, SD 5.37; SEM (show on figure) is 0.08 . See Table 5 for a comparison of the mean of repetitions completed without burn in each of the conditions, Control vs. Supplemented, for squat, deadlift, and floor press.

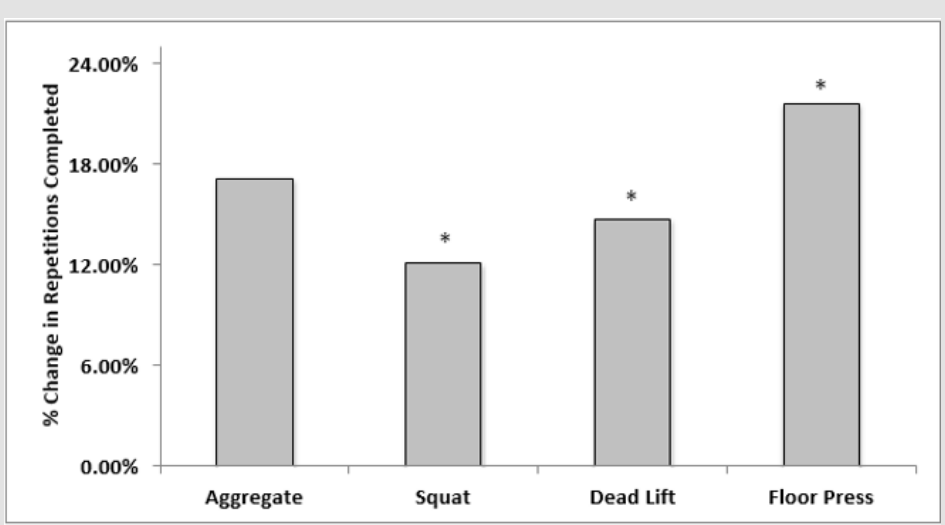

Figure 5: \% Improvement in mean repetitions completed without burn in the Supplemented condition. Significantly different. For $\mathrm{p}$ values see Table 5 . 
For the squat, out of the 140 sets completed in the Supplemented condition, 82 (59\%) sets had a delay in the onset of burn during the sets completed, 44 (31\%) sets presented a hasted onset of burn, while $14(10 \%)$ sets presented no change in the onset of burn moment. Similarly, the variance in deadlift repetitions completed at which athletes reported the onset of burn between the Control and Supplemented condition was significant ( $p \leq 0.05$ ). Out of the 140 sets completed, in the Supplemented condition, 77 (55\%) sets had a delay in the onset of burn during the sets completed, 41 (29\%) sets presented a hasted onset of burn, while 22 (16\%) sets presented no change in the onset of burn moment. Finally, for the floor press, the variance in repetitions completed at which athletes reported the onset of burn between the Control and Supplemented condition was also significant ( $\mathrm{p} \leq 0.05)$. Out of the 140 sets completed, in the Supplemented condition, 95 (68\%) sets had a delay in the onset of burn during the sets completed, 28 (20\%) sets presented a hasten onset of burn, while $17(12 \%)$ sets presented no change in the onset of burn moment.

\section{EWC - Peak Burn Severity (PBS)}

There appears to be a significant impact $(\mathrm{p}<0.01)$ on the selfreported peak burn severity (Tables 6- 10 \& Figure 6-8). Under Supplemented conditions, athletes self-reported a reduction in the peak severity of exercise induced muscle burn.

Table 6: Change in PBS in Control vs. Supplemented Conditions $\left({ }^{*} \mathrm{p}<0.01\right)$.

\begin{tabular}{|c|c|c|c|}
\hline Burn Severity & Control & Supplemented & Change * \\
\hline $1-$ no burn & 66 & 105 & 39.00 \\
\hline $2-$ onset & 71 & 113 & 42.00 \\
\hline $3-$ mild & 144 & 111 & -33.00 \\
\hline $4-$ moderate & 110 & 82 & -28.00 \\
\hline $5-$ severe & 26 & 8 & -18.00 \\
\hline
\end{tabular}

Table 7: Change Reported in PBS in Control vs. Supplemented Conditions; Squats. $\left({ }^{*} \mathrm{p}<0.01\right)$.

\begin{tabular}{|c|c|c|c|c|}
\hline & Control & Supplemented & Change & \% Change * \\
\hline $1-$ no burn & 21 & 37 & 16.00 & $76.19 \%$ \\
\hline 2 - onset & 33 & 43 & 10.00 & $30.30 \%$ \\
\hline 3 - mild & 54 & 45 & -9.00 & $-16.67 \%$ \\
\hline $4-$ moderate & 25 & 15 & -10.00 & $-40.00 \%$ \\
\hline $5-$ severe & 5 & 0 & -5.00 & $-100.00 \%$ \\
\hline
\end{tabular}

Table 8: Change Reported in PBS in Control vs. Supplemented Conditions; Deadlifts. $\left({ }^{*} \mathrm{p}<0.01\right)$.

\begin{tabular}{|c|c|c|c|c|}
\hline & Control & Supplemented & Change & \% Change * \\
\hline 1 - no burn & 26 & 40 & 14.00 & $53.85 \%$ \\
\hline 2 - onset & 13 & 40 & 27.00 & $207.69 \%$ \\
\hline 3 - mild & 46 & 30 & -16.00 & $-34.78 \%$ \\
\hline $4-$ moderate & 49 & 26 & -23.00 & $-46.94 \%$ \\
\hline 5 - severe & 4 & 1 & -3.00 & $-75.00 \%$ \\
\hline
\end{tabular}

Table 9: Change reported in PBS in Control vs. Supplemented Conditions; Floor Press. (*p < 0.01).

\begin{tabular}{|c|c|c|c|c|}
\hline & Control & Supplemented & Change & \% Change * \\
\hline 1 - no burn & 19 & 28 & 9.00 & $47.37 \%$ \\
\hline 2 - onset & 24 & 29 & 5.00 & $20.83 \%$ \\
\hline 3 - mild & 42 & 34 & -8.00 & $-19.05 \%$ \\
\hline $4-$ moderate & 35 & 39 & 4.00 & $11.43 \%$ \\
\hline 5 - severe & 17 & 7 & -10.00 & $-58.82 \%$ \\
\hline
\end{tabular}

Table 10: "SD" is the Standard Deviation; "SEM" is the Standard Error of the Mean. Change is Significant for Squat d Deadlift ("p < $0.01)$. Change is not significant for Floor Press ( $¥ p \leq 0.06)$.

\begin{tabular}{|c|c|c|c|c|}
\hline \multicolumn{5}{|c|}{ Change in Reported PBS For Squat, Deadlift, and Floor Press } \\
\hline & Control PBS (SD, SEM) & Supplemented PBS (SD, SEM) & Change & p \\
\hline Squat & $2.72(1.04,0.05)$ & $2.27(0.97,0.05)$ & $-0.45 \pm .14$ & $<0.01$ \\
\hline Deadlift & $2.94(1.14,0.06)$ & $2.35(1.11,0.06)$ & $-0.59 \pm .18$ & $<0.01$ \\
\hline Floor Press & $3.03(1.24,0.06)$ & $2.75(1.23,0.06)$ & $-0.28 \pm .15$ & $\leq 0.06$ \\
\hline
\end{tabular}




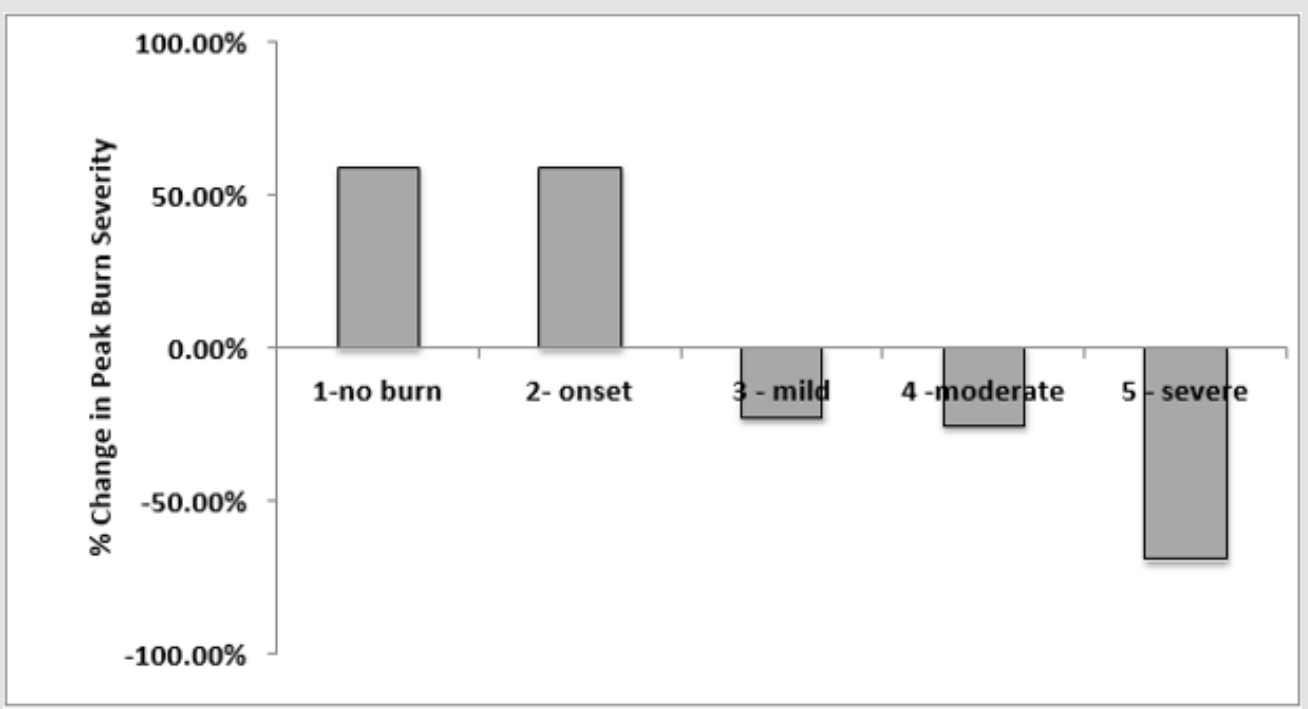

Figure 6: Under Supplemented conditions aggregate reported PBS is reduced. Changes are significant $(\mathrm{p}<0.01)$.

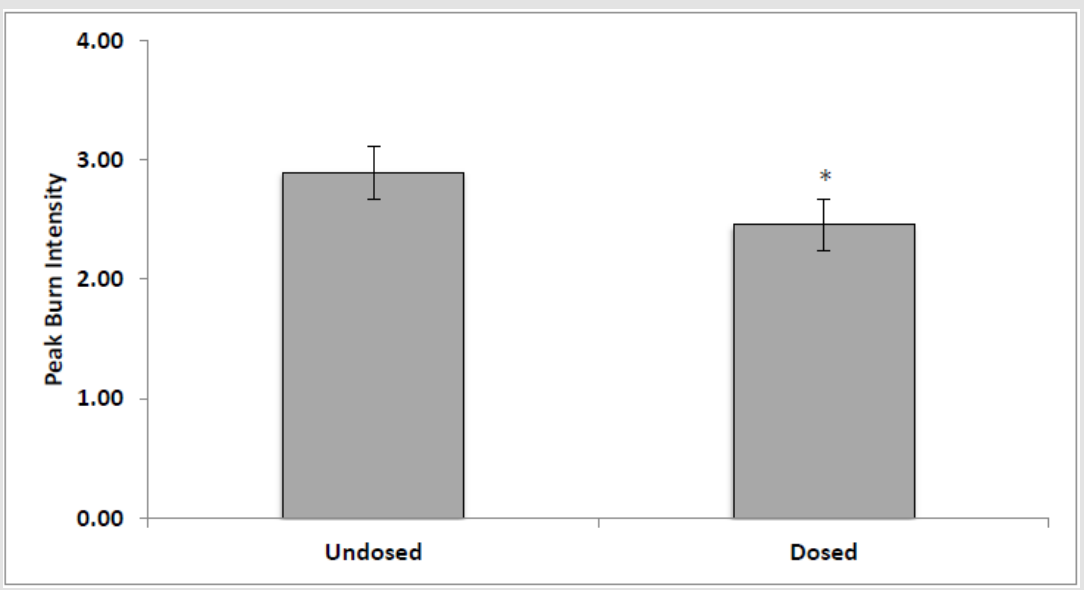

Figure 7: Mean change in reported PBS decreased by $0.44 \pm .09$ in the Supplemented condition vs. the Control condition. Significantly different $\left({ }^{*} \mathrm{p}<0.01\right)$. Control mean 2.90, SD is 1.15 (Excel stdev.p); SEM (shown on figure) is 0.03. Supplemented mean 2.46, SD 1.13 (Excel stdev. p); SEM (shown on figure) is $\mathrm{p} \leq 0.04$.

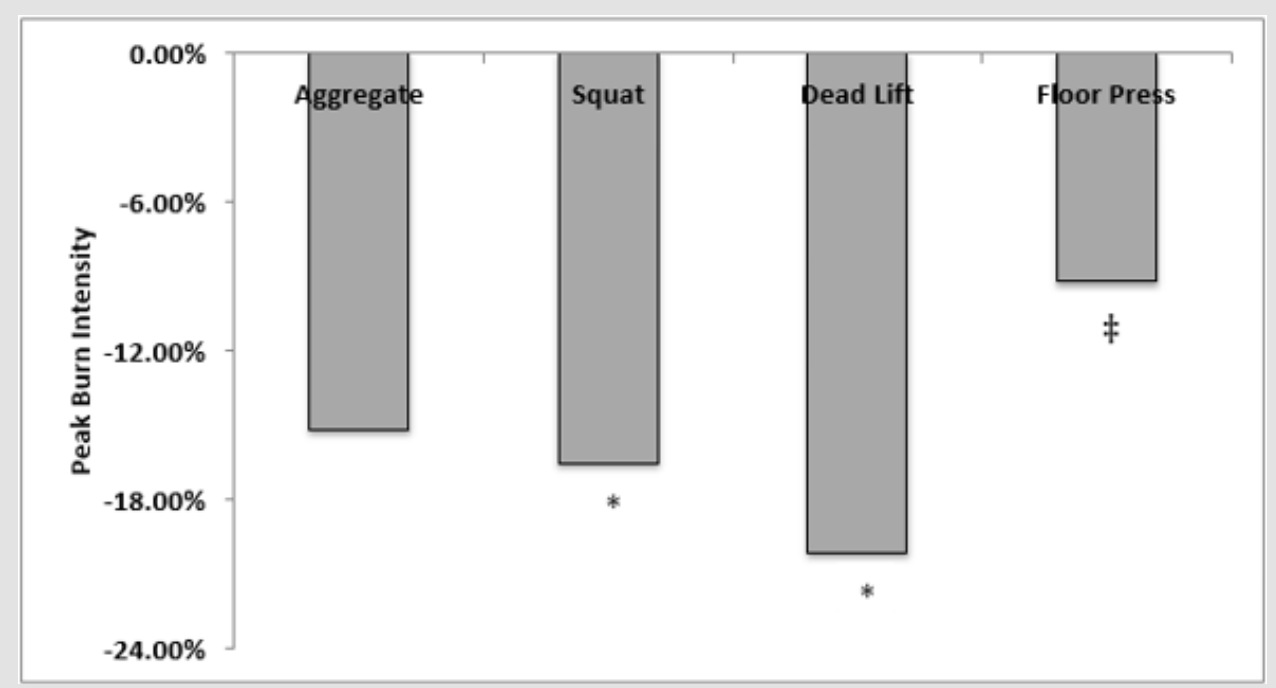

Figure 8: Percent reduction in mean PBS experienced in the Supplemented condition. ${ }^{*} \mathrm{p} \leq 0.01, \ddagger \mathrm{p} \leq 0.06$. 


\section{Discussion}

A summary of the variance in the aggregate repetitions in Supplemented condition is significantly positive with an $8.5 \%$ increase in volume of work due to supplementation without any specific training for a determined period of time. While, studies investigated the optimal zone for volume and intensity to produce significant effect on increased physical capacity, we chose to eliminate those factors and see if the nutritional intervention produces efficient increases in physical performance. The literature is abundant with studies of training modulation from intensity or/and volume perspectives compiled or not with a nutrition component. Katmandulis et al. [53] looked at 8 weeks of higher frequency of training. The effect produced in regard to legs muscle strength was of $1.5 \%$, improvements like those found by Lester et al., [54] which demonstrated greater gains in strength with a higher frequency training regimen. Thomas and Burns [55] identified an 11\% increase in strength after 8 weeks of high frequency strength training and $7 \%$ for the low frequency group where the two groups were not statistically different, so the results were equivocal. As seen in Table 3, a sufficient frequency and volume of work regardless of the mode (different muscle actions: dynamic external resistance, isokinetic devices, isometric effort) are needed to increase muscle gains.

A study by Mangine, et. al. [56] determined that increased volume of work and not the frequency is the essential factor for hypertrophy and developing strength in men during a resistance program, which is a pivotal step in sports training and rehabilitation. Although the research sometimes cannot identify "the main" single factor to influence performance, volume is one of the essential aspects in improving athletic performance. Improved ability to complete higher volume workouts will not only increase strength it will also be a factor in maintaining high level of technical and tactical performance for a longer period of time (sport specific endurance) $[57,58]$. Eliminating the modulation of intensity, frequency factor as well as the specific training factor during a certain prescribed period of time this study isolates the effects of supplementation on our participants. Without any specific preparation between the Control and Supplemented workouts and with a time frame of only two weeks, between the Control and Supplemented session, we used nutritional intervention and achieved a significant change in volume of work through an increase in repetitions completed (from 4942 repetitions to 5429 repetitions per aggregate), without altering the weight.

This study demonstrated that a $17 \%$ increase in repetitions completed without signs of exercise induced muscle soreness was related to an $8.5 \%$ improvement in the volume of work. Statistically there is a definite correlation between the two afore mentioned aspects of work capacity. The moment of the "onset of burn" signaled with an average of a repetition later in the Supplemented condition compared to the Control condition also influenced the improvement in the average aggregates performance by approximately one repetition (mean improvement in repetitions completed from 11.77 repetitions to 12.93 repetitions). Temporary, as it is known, repairable muscle damage is often caused by the novelty of exercise, which can generate metabolic disturbances associated with ischemia. Our volunteers have not performed a workout in this format before our study. Exercise induced muscle damage, which is associated with the self-reported muscle burn results in a temporary decrease in muscle force production and an increase in soreness. Researchers, coaches and athletes have been preoccupied to investigate, reduce and curb the side effects of muscle soreness as studies have been registering a significant decrease in athletic performance. Marcora et al. [59] recorded a reduced running speed by $4 \%$ in a self-paced trial due to muscle soreness. With increased perceived exertion and decreased time of performance it was concluded that exercise muscle soreness has a negative impact on running endurance.

Another exercise modality was investigated, and it has been established that after a plyometric muscle damaging exercise protocol, the muscle's ability to produce power is reduced for at least three days. Thus, there is a consensus regarding the negative effect that the exercise muscle soreness has upon athletic performance. Our data, along with other investigative efforts, come to establish the connection between work capacity and muscle soreness and offers a possible solution to increase performance through supplementation. For example, one dose of citrulline malate resulted in a $52.92 \%$ improvement in flat bench press repetitions and a $40 \%$ decrease muscle soreness (citrulline malate decrease soreness [60]. One dose of malate citrulline, components of our nutritional interventions increases performance and decreased DOMS in high intensity exercise with short rest times (citrulline malate enhances athletic anaerobic performance and relieves muscle soreness[30]). Malate stimulates tricarboxylic acid intermediates (TCAI) pool and the overall flux within the Krebs cycle, which both can be regarded as means to attaining higher rates of aerobic energy production and as a result a reduction in fatigue sensation reported by the subjects (citrulline malate promotes aerobic energy production inhuman exercising muscle - [30]). Our research findings come to aid the athletic community in order to choose the best type of training interventions according to the specifics of the physical activity and the performance goals established.

Using research results, most of the time coaches, athletes and medical staff have used supplements to curb the effects of muscle soreness on performance and prevent injuries. Multiple recovery options such as electrical therapy and cryotherapy and other interventions offer limited effect in the treatment of exercise induced muscle soreness [61]. The diversity of recommendations regarding dosage and frequency of these recovery methods could be the culprit for not reaching a consensus in terms of their efficacy. Compared to the incidence of muscle soreness phenomenon in physical exercise there are very few evidence-based guidelines of how these methods should be applied to reach their full potential [62-66]. The aggregate, under the Supplemented conditions, 
experienced $15.2 \%$ decrease in muscle burn and athletes experiencing only eight instances of impaired form and severe level of soreness. The muscle fibers undergoing workout related stress could be eliminated or regenerate along with the changes suffered by the connective tissue and offer a greater resistance to further stimuli. Concordantly, studies have shown that supplementing mice with L-malate improved the effort time from $21.1 \%$ to $28 \%$. This result can be used as predictor for L-malate improving physical stamina and minimizing muscle damage [67].

Vitamin C, which is a component of the nutritional intervention strategy appears to offer protection against muscle damage and may also improve muscle function. The purported potential of Vitamin C for reducing inflammation within muscle tissue [68], may explain the diminished muscle soreness (Vitamin $\mathrm{C}$ as an ergogenic aid) in the Supplemented condition. This study functionally represents a pilot investigation into the possible efficacy of nutritional intervention directed at improving athletic performance and recovery [69]. As such, we have not used a placebo group at this time, and we considered the parameters considered within this study to be stable and reproducible. It is recommendable that prior to the study, the volunteers should participate in 1RM training session opposed to establish our calculation according to the selfreported numbers. We can infer according to the instances of 1 and 2 level of experienced muscle pain (burn), that we would have had a more dramatic change in the peak burn severity between Control and Supplemented condition. We had a high number of number 1 instances in PBS and considering the high volume and intensity pace, we can only conclude that 1RM was likely not very accurate for all participants.

The nutritional intervention although it was very controlled from a time point of view, it should have been better customized according to the weight of the participants. Although the intent of the dosage was to emulate real life conditions, given the design of the study and the fact that the volunteers were evaluated in their performance being compared against themselves, we consider the results accurate despite the limitations in this pilot study. The measured improvements during the Supplemented condition demonstrate that the nutritional intervention used (pyruvate, malate, citrate, Vitamin C) increases exercise work capacity without specific training being developed and it is efficacious relatively fast to improve both performance and recovery.

The protocol designed for this study can also be used as a testing method of basic strength in the general conditioning phase of a macrocycle. As athletes are looking for different methods of performance enhancement that could make the difference at highly competitive level, our proposed nutritional intervention could have an impact on their result as it was proven in this study as having a major positive influence on physical exertion and ability to sustain it at high levels. The increased volume came as a result of a delayed onset of burn. The prolongation of pain free performance time coupled with the reduced peak burn severity generated a greater work capacity. Because the work time during BDP was time-capped and supplementation was added, the onset of burn happened later than the Control condition. Therefore, the fatigue installed later, and the participants were able to execute more repetitions overall. Because the onset of burn happened later during the working sets, the peak severity didn't reach the same level in the time allocated to complete the set. The supplementation had a major influence on all these factors and the result was a definite enhancement in athletic performance. It remains to be investigated if the nutritional intervention will have the same impact on an anaerobic effort with declining rest or on an aerobic type of effort.

\section{Acknowledgement}

The authors would like to express their thanks and gratitude to Ms. Teresa Artuso for her diligence in compiling the manuscript, the members of Seneca College's Division of Applied Research, Innovation and Entrepreneurship (ARIE), and the support of Seneca's Departmental Chair, Ms. Paola Battiston, for her continuous support of research activities at Seneca College. We appreciate and are grateful to Seneca's applied research fund (ARF) awarded to F. Merante, whose kind funding permitted this body of work to be completed.

\section{References}

1. Armstrong RB, Warren GL, Warren JA (1991) Mechanisms of exerciseinduced muscle fiber injury. Sports Med 12(3): 184-207.

2. Ebbeling CB, Clarkson PM (1989) Exercise-Induced Muscle Damage and Adaptation. Sports Med 7(4): 207-234.

3. J Fridén, M Sjöström, B Ekblom (1983) Myofibrillar Damage Following Intense Eccentric Exercise in Man. Int J Sports Med 04(3): 170-176.

4. Marcora SM, Bosio A (2007) Effect of exercise induced muscle damage on endurance running performance in humans. Med Scien Sports 17(6): 662-671.

5. Cheung K, Hume PA, Maxwell P (2003) Delayed onset muscle soreness. Sports Med 33(2): 145-164.

6. Bendahan D, Mattei JP, Ghattas B, Confort Gouny S, Le Guern ME, et al. (2002) Citrulline/malate promotes aerobic energy production in human exercising muscle. Br J Sports Med 36(4): 282-289.

7. Bruce M, Constantin Teodosiu D, Greenhaff PL, Boobis LH, Williams C, et al. (2001) Glutamine supplementation promotes anaplerosis but not oxidative energy delivery in human skeletal muscle. Am J Physiol Endocrinol Metab 280(4): E669-675.

8. Heinonen OJ (1996) Carnitine and physical exercise. Sports Med 22(2): 109-132.

9. Shan L, Wang B, Gao G, Cao W, Zhang Y, et al. (2013) L-Arginine supplementation improves antioxidant defenses through L-arginine/ nitric oxide pathways in exercised rats. J Appl Physiol (1985) 115(8): 1146-1155.

10. Hobson R M, Saunders B, Ball G, Harris R C, C Sale, et al. (2012) Effects of $\beta$-Alanine supplementation on exercise performance: a meta-analysis. Amino Acids 43 (1): 25-37.

11. Hobson RM, Saunders B, Ball G, Harris RC, Sale C (2012) Effects of $\beta$-alanine supplementation on exercise performance: a meta-analysis. Amino Acids 43(1): 25-37.

12. Wax B, Kavazis AN, Luckett W (2016) Effects of Supplemental Citrulline Malate Ingestion on Blood Lactate Cardiovascular Dynamics and 
Resistance Exercise Performance in Trained Males. J Diet Suppl 13(3): 269-282.

13. Wax B, Kavazis AN, Weldon K, Sperlak J (2015) Effects of supplemental citrulline malate ingestion during repeated bouts of lower-body exercise in advanced weightlifters. J Strength Cond Res 29(3): 786-792.

14. Pérez Guisado J, Jakeman PM (2010) Citrulline malate enhances athletic anaerobic performance and relieves muscle soreness. J Strength Cond Res 24(5): 1215-1222.

15. Takeda K, Machida M, Kohara A, Omi N, Takemasa T, et al. (2011) Effects of citrulline supplementation on fatigue and exercise performance in mice. J Nutr Sci Vitaminol (Tokyo) 57(3): 246-250.

16. Wax B, Kavazis AN, Luckett W (2016) Effects of Supplemental Citrulline Malate Ingestion on Blood Lactate Cardiovascular Dynamics and Resistance Exercise Performance in Trained Males. J Diet Suppl 13(3): 269-282.

17. Ishikura K, Miyazaki T, Ra SG, Endo S, Nakamura Y, et al. (2011) Effect of taurine supplementation on the alterations in amino Acid content in skeletal muscle with exercise in rat. J Sports Sci Med 10(2): 306-314.

18. Williams M (2005) Dietary Supplements and Sport Performance: Amino Acids. J Int Soc Sports Nutr. 2(2): 63-67.

19. Crowe MJ, Weatherson JN, Bowden BF (2006) Effects of dietary leucine supplementation on exercise performance. Eur J Appl Physiol 97(6): 664-672.

20. She P, Zhou Y, Zhang Z, Griffin K, Gowda K, et al. (2010) Disruption of BCAA metabolism in mice impairs exercise metabolism and endurance. J Appl Physiol (1985) 108(4): 941-949.

21. Crowe M J, Weatherson J N, Bowden B F (2006) Effects of dietary leucine supplementation on exercise performance. Euro J of App Physio 97 (6): 664-672.

22. Chang CK, Chang Chien KM, Chang J H, Huang MH, Liang YC, et al. (2015) Branched-chain Amino Acids and Arginine Improve Performance in Two Consecutive Days of Simulated Handball Games in Male and Female Athletes: A Randomize Trial. J Plos 10(3): e0121866.

23. Ivy JL (1998) Effect of pyruvate and dihydroxyacetone on metabolism and aerobic endurance capacity. Med Sci Sports Exerc 30(6): 837-843.

24. Kalman D, Colker CM, Stark R, Minsch A, Wilets I, et al. (1999) Effect of pyruvate supplementation on body composition and mood. Current Therapeutic Research 59(11): 793-802.

25. Stone MH, Sanborn K, Smith LL, O Bryant HS, Hoke T, et al. (1999) Effects of in-season (5 weeks) creatine and pyruvate supplementation on anaerobic performance and body composition in American football players. Int J Sport Nutr 9(2): 146- 165.

26. Russell C, Papadopoulos E, Mezil Y, Wells GD, Plyley MJ, et al. (2014) Acute versus chronic supplementation of sodium citrate on $200 \mathrm{~m}$ performance in adolescent swimmers. J Int Soc Sports Nutr 11: 26.

27. Timpmann S, Burk A, Medijainen L, Tamm M, Kreegipuu K, et al. (2012) Dietary sodium citrate supplementation enhances rehydration and recovery from rapid body mass loss in trained wrestlers. Appl Physiol Nutr Metab 37(6): 1028-1037.

28. Oopik V, Saaremets I, Medijainen L, Karelson K, Janson T, et al. (2003) Effects of sodium citrate ingestion before exercise on endurance performance in well trained college runners. Br J Sports Med 37(6): 485-489.

29. Wu JL, Wu QP, Huang JM, Chen R, Cai M, et al. (2007) Effects of L-malate on physical stamina and activities of enzymes related to the malate aspartate shuttle in liver of mice. Physiol Res 56(2): 213- 220.

30. Buford T W, Kreider R B, Stout J R, Greenwood M, Campbell B, et al. (2007) International Society of Sports Nutrition position stand: creatine supplementation and exercise. JISSN 4(6).

31. Chwalbinska Moneta J (2003) Effect of creatine supplementation on aerobic performance and anaerobic capacity in elite rowers in the course of endurance training. Int J Sport Nutr Exerc Metab 13 (2): 173-183.
32. Jäger R, Metzger J, Lautmann K, Shushakov V, Purpura M, et al. (2008) The effects of creatine pyruvate and creatine citrate on performance during high intensity exercise. J Int Soc Sports Nutr 5: 4.

33. Tyka AK, Chwastowski M, Cison T, Palka T, Tyka A, et al. (2015) Effect of creatine malate supplementation on physical performance body composition and selected hormone levels in sprinters and long-distance runners. Acta Physiol Hung 102(1): 114-122.

34. Heinonen OJ (1996) Carnitine and physical exercise. Sports Med 22(2): 109-132.

35. Wall BT, Stephens FB, Constantin Teodosiu D, Marimuthu K, Macdonald IA, et al. (2011) Chronic oral ingestion of L-carnitine and carbohydrate increases muscle carnitine content and alters muscle fuel metabolism during exercise in humans. J Physiol 589(Pt 4): 963-973.

36. Van der Beek E J (1991) Vitamin supplementation and physical exercise. J Sport Sci 9(1): 77-89.

37.Van der Beek EJ, Vitamin supplementation and physical exercise performance. J Sports Sci 9: 77-90.

38. Mizuno K, Tanaka M, Nozaki S, Mizuma H, Ataka S, et al. (2008) Antifatigue effects of coenzyme Q10 during physical fatigue. Nutri 24(4): 293-299.

39. Rosa EF, Ribeiro RF, Pereira FM, Freymüller E, Aboulafia J, et al. (2009) Vitamin $\mathrm{C}$ and $\mathrm{E}$ supplementation prevents mitochondrial damage of ileum myocytes caused by intense and exhaustive exercise training. J Appl Physiol 107(5): 1532-1538.

40. Rosa EF, Ribeiro RF, Pereira FM, Freymüller E, Aboulafia J, et al. (2009) Vitamin C and E supplementation prevents mitochondrial damage of ileum myocytes caused by intense and exhaustive exercise training. J Appl Physiol 107(5): 1532-1538.

41. Hodgson AB, Randell RK, Boon N, Garczarek U, Mela DJ, et al. (2013) Metabolic response to green tea extract during rest and moderateintensity exercise. J Nutr Biochem 24(1): 325-334.

42. Herda TJ, Ryan ED, Stout JR, Cramer JT (2008) Effects of a supplement designed to increase ATP levels on muscle strength power output and endurance. J Int Soc Sports Nutr 5: 3.

43. Currell K, Syed A, Dziedzic CE, King DS, Spriet LL, et al. (2010) A- Z of nutritional supplements: dietary supplements, sports nutrition foods and ergogenic aids for health and performance--part 12. Br J Sports Med 44(12): 905-907.

44. Vukovich MD, Sharp RL, King DS, Kershishnik K (1992) The effect of protein supplementation on lactate accumulation during submaximal and maximal exercise. Int J Sport Nutr 2(4): 307-316.

45. Yavuz H U, Turnagol H, Demirel A H (2014) Pre-exercise arginine supplementation increases time to exhaustion in elite male wrestlers. Biol Sport 3(1): 187-191.

46. Yavuz HU, Turnagol H, Demirel AH (2014) Pre exercise arginine supplementation increases time to exhaustion in elite male wrestlers. Biol Sport 31(3): 187-191.

47. Fouré A, Nosaka K, Gastaldi M, Mattei JP, Boudinet H, et al. (2015) Effects of branched-chain amino acids supplementation on both plasma amino acids concentration and muscle energetics changes resulting from muscle damage: A randomized placebo-controlled trial. Clin Nutr 35(1): 83-94.

48. Manjarrez Montes de Oca R, Farfán González F, Camarillo Romero S, Tlatempa Sotelo P, Francisco Argüelles C, et al. (2013) Effects of creatine supplementation in taekwondo practitioners. Nutr Hosp 28(2): 391399.

49. Chang CK, Chang Chien KM, Chang JH, Huang MH, Liang YC, et al. (2015) Branched-chain amino acids and arginine improve performance in two consecutive days of simulated handball games in male and female athletes: a randomized trial. PLoS One 10(3): e0121866.

50. Wesselink E, Koekkoek WAC, Grefte S, Witkamp RF, van Zanten ARH, et al. (2019) Feeding mitochondria: Potential role of nutritional components to improve critical illness convalescence. Clin Nutr 38(3): 982-995. 
51. Rinninella E, Pizzoferrato M, Cintoni M, Servidei S, Mele MC, et al. (2018) Nutritional support in mitochondrial diseases: the state of the art. Eur Rev Med Pharmacol Sci 22(13): 4288-4298.

52. Leite HP, De Lima LF (2016) Metabolic resuscitation in sepsis: a necessary step beyond the hemodynamic? J Thorac Dis 8(7): E552-557.

53. Kamandulis S, Skurvydas A, Brazaitis M, Stanislovaitis A, Duchateau J, et al. (2012) Effect of a periodized power training program on the functional performances and contractile properties of the quadriceps in sprinters. Res Q Exerc Sport 83(4): 540-545.

54. Howatson G, Van Someren KA (2008) The Prevention and Treatment of Exercise-Induced Muscle Damage. Sports Med 38 (6): 483-503.

55. Crowe M J, Weatherson J N, Bowden B F (2006) Effects of dietary leucine supplementation on exercise performance. Euro J of App Physio 97 (6): 664-672.

56. Mangine GT, Hoffman JR, Gonzalez AM, Townsend JR, Wells AJ, et al. (2015) The effect of training volume and intensity on improvements in muscular strength and size in resistance-trained men. Physiol Rep 3(8): e12472.

57. Van der Beek E J (1991) Vitamin supplementation and physical exercise. J Sport Sci 9(1): 77-89.

58. Yavuz H U, Turnagol H, Demirel A H (2014) Pre-exercise arginine supplementation increases time to exhaustion in elite male wrestlers. Biol Sport 3(1): 187-191.

59. Marcora SM, Bosio A (2007) Effect of exercise induced muscle damage on endurance running performance in humans. Scand J Med Sci Sports 17(6): 662-671.

60. Twist C, Eston R (2005) The effects of exercise-induced muscle damage on maximal intensity intermittent exercise performance. Eur J Appl Physio 94(5-6): 652-658.

\section{ISSN: 2574-1241}

DOI: $10.26717 /$ BJSTR.2019.23.003944

Frank Merante. Biomed J Sci \& Tech Res

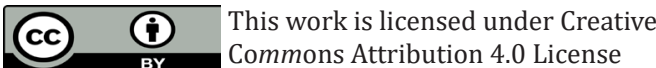

Submission Link: https://biomedres.us/submit-manuscript.php
61. Paschalis V, Koutedakis Y, Jamurtas AZ, Mougios V, Baltzopoulos V, et al. (2005) Equal volumes of high and low intensity of eccentric exercise in relation to muscle damage and performance. J Strength Cond Res 19(1): 184-188.

62. Marco C Uchida, Ken Nosaka, Carlos Ugrinowitsch, Alex Yamashita, Eivor Martins Jr, et al. (2009) Effect of bench press exercise intensity on muscle soreness and inflammatory mediators. J Sports Scien 27(5): 499-507.

63. Candow D G, Burke D G (2007) Effect of short term equal volume resistance training with different workout frequency on muscle mass and strength in untrained men and women. J of Strg and Cond Rsch 21(1): 204-207.

64. Willardson JM, Burkett LN (2005) A comparison of 3 different rest intervals on the exercise volume completed during a workout. J Strength Cond Res 19(1): 23-26.

65. Rahman R (2005) Effect of Different Rest Intervals on the Exercise Volume Completed During Squat Bouts. J Sports Sci Med 4(4): 361-366.

66. Lester ME, Sharp MA, Werling WC, Walker LA, Cohen BS, et al. (2014) Effect of specific short- term physical training on fitness measures in conditioned men. J Strength Cond Res 28(3): 679-688.

67. Thomas MH, Burns SP (2016) Increasing Lean Mass and Strength: A Comparison of High Frequency Strength Training to Lower Frequency Strength Training. Int J Exerc Sci 9(2): 159-167.

68. Wu JL, Wu QP, Huang JM, Chen R, Cai M, et al. (2007) Effects of L malate on physical stamina and activities of enzymes related to the malateaspartate shuttle in liver of mice. Physiol Res 56(2): 213-220.

69. Bailey DM, Williams C, Betts JA, Thompson D, Hurst TL, et al. (2011) Oxidative stress, inflammation and recovery of muscle function after damaging exercise: effect of 6-week mixed antioxidant supplementation. Eur J Appl Physiol 111(6): 925-936.

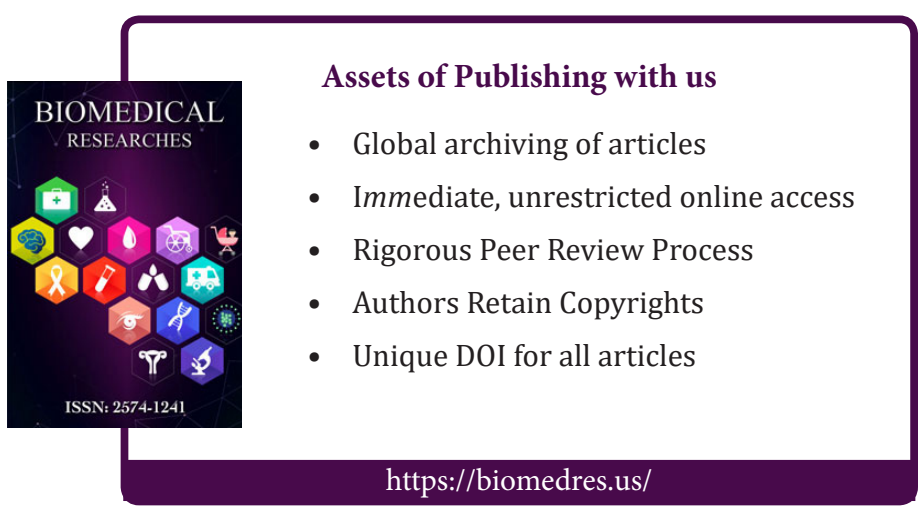

